\title{
(6) OPEN ACCESS \\ Evidence-based approaches to childhood stunting in low and middle income countries: a systematic review
}

\author{
Muttaquina Hossain, ${ }^{1}$ Nuzhat Choudhury, ${ }^{1}$ Khaleda Adib Binte Abdullah, ${ }^{1}$ \\ Prasenjit Mondal, ${ }^{1}$ Alan A Jackson, ${ }^{2}$ Judd Walson, ${ }^{3}$ Tahmeed Ahmed ${ }^{1}$
}

\begin{abstract}
- Additional material is published online only. To view please visit the journal online (http://dx.doi.org/10.1136/ archdischild-2016-311050).

${ }^{1}$ Stunting Research Platform, Nutrition and Clinical Services Division, ICDDR, Dhaka, Bangladesh

${ }^{2}$ Southampton General Hospital, Southampton, UK

${ }^{3}$ Department of Global Health, University of Washington, Seattle, USA
\end{abstract}

Correspondence to Dr Muttaquina Hossain, Nutrition and Clinical Services Division, International Centre for Diarrhoeal Disease Research, 68 Shahid Tajuddin Ahmed Sarani, Mohakhali, Dhaka 1212, Bangladesh;

muttaquina@icddrb.org

Received 18 April 2016 Revised 21 September 2016 Accepted 2 December 2016

Published Online First 27 April 2017

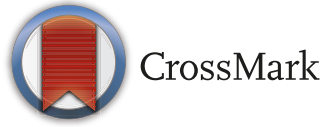

To cite: Hossain M, Choudhury N, Adib Binte Abdullah K, et al.

Arch Dis Child

2017;102:903-909.

\section{ABSTRACT}

Objective We systematically evaluated health and nutrition programmes to identify context-specific interventional packages that might help to prioritise the implementation of programmes for reducing stunting in low and middle income countries (LMICS).

Methods Electronic databases were used to systematically review the literature published between 1980 and 2015. Additional articles were identified from the reference lists and grey literature. Programmes were identified in which nutrition-specific and nutritionsensitive interventions had been implemented for children under 5 years of age in LMICs. The primary outcome was a change in stunting prevalence, estimated as the average annual rate of reduction (AARR). A realist approach was applied to identify mechanisms underpinning programme success in particular contexts and settings.

Findings Fourteen programmes, which demonstrated reductions in stunting, were identified from 19 LMICs. The AARR varied from 0.6 to 8.4. The interventions most commonly implemented were nutrition education and counselling, growth monitoring and promotion, immunisation, water, sanitation and hygiene, and social safety nets. A programme was considered to have effectively reduced stunting when $A A R R \geq 3 \%$.

Successful interventions were characterised by a combination of political commitment, multi-sectoral collaboration, community engagement, communitybased service delivery platform, and wider programme coverage and compliance. Even for similar interventions the outcome could be compromised if the context differed.

Interpretation For all settings, a combination of interventions was associated with success when they included health and nutrition outcomes and social safety nets. An effective programme for stunting reduction embraced country-level commitment together with community engagement and programme context, reflecting the complex nature of exposures of relevance.

PROSPERO registration number CRD42016043772.

\section{INTRODUCTION}

Stunting ${ }^{1}$ is the most common manifestation of chronic undernutrition during childhood in low and middle income countries (LMICs). It has been associated with increased risk of morbidity and
What is already known on this topic?

- A combination of nutrition-specific and nutrition-sensitive interventions appears necessary in order to optimise programmes to reduce stunting.

\section{What this study adds?}

- Nutrition education and counselling, growth monitoring and promotion, immunisation, water, sanitation and hygiene and social safety net programmes appear to be the most commonly included interventions of an effective package in most low and middle income countries settings.

- Single interventions reduced stunting only in countries with specific disease burden.

- Intervention worked best when country, community and programme context were taken into account.

mortality and impacts negatively on both physical growth and cognitive development; as well as increased risk of obesity and other metabolic complications in later life. Separately and together these lead to lower economic productivity, and constrained social function. ${ }^{2}$ Thus, stunting appears a direct impediment towards achieving the sustainable development goals.

The most vulnerable period for the establishment of lifelong stunting is the first 1000 days from conception. Thereafter, it appears increasingly difficult to reverse adverse influences ultimately leading to adults of shorter stature. ${ }^{3}$ In targeting the first 1000 days, both global movements and regional programmes have sought to take nutrition interventions to scale by supporting nutrition governance. ${ }^{4}$ However, these initiatives appear less successful than anticipated in achieving the desired reduction in stunting in LMICs. ${ }^{5}$

Globally, stunting has decreased at approximately $1.8 \%$ per year. $^{6}$ indicating that it will only decrease by $18 \%$ in the next 10 years, failing to achieve the goal set by the World Health Assembly. ${ }^{7}$ This is consistent with data suggesting that scaling up 
Figure 1 Definition of nutrition-specific and nutrition-sensitive interventions and programmes (adapted from Ruel and Alderman $\left.{ }^{9}\right)$.

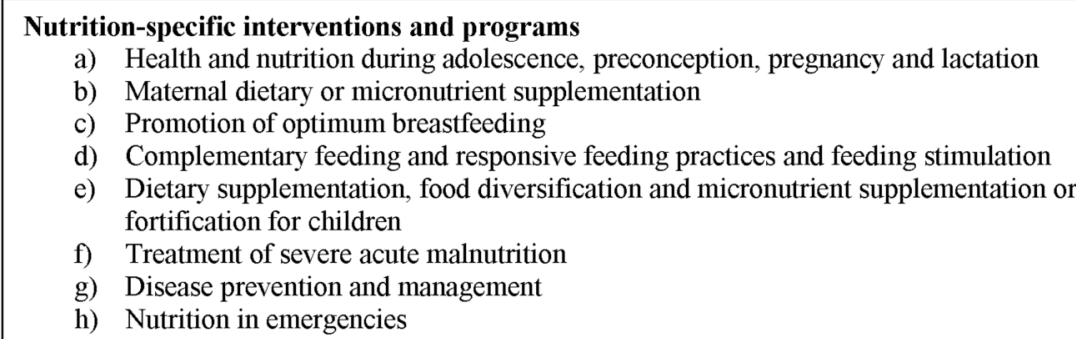

Nutrition-sensitive interventions and programs

a) Agriculture and food security

b) Social safety nets

c) Early child development

d) Maternal mental health

e) Women's empowerment

f) Child protection

g) Schooling

h) Water, sanitation and hygiene

i) Health and family planning services existing nutrition-specific interventions would reduce stunting by about $20 \% .{ }^{8}$ A combination of nutrition-specific and nutrition-sensitive interventions appears necessary in order to optimise reductions in stunting (figure 1). ${ }^{9}$

This review sought to identify studies from LMICs where combined programmatic interventions have been evaluated. It identifies the programmes and the components of nutrition interventions in which there had been demonstrable success leading to a reduction in stunting. In addition, we sought to determine correlates of success among programmes for particular contexts and settings by using the realist approach. The purpose was to provide a synthesis of the available evidence that could assist policy makers and donors in prioritising the use of resources for implementation of interventions to prevent and control stunting in resource limited settings by considering of a mix of factors for the country, community and programme contexts.

\section{METHODS}

\section{Data sources}

A comprehensive search strategy was designed a priori and applied to electronic bibliographic databases, including Medline (Pub Med), WHO Regional databases, Google's Scholar databases and the Cochrane Library with specific key words/concepts: 'stunting', 'linear growth failure', 'stunting reduction programme', 'intervention', 'approach' and 'low and middle income countries' (see online supplementary appendix 1). The search was limited to literature published between 1 January 1980 and 31 March 2015. MeSH headings were used where available. Published and unpublished references and grey literature sources were searched electronically and manually.

\section{Study eligibility criteria}

This review considered all studies involving human subjects under 5 years of age (0-59 months), published in the English language. It had a focus on public health programmes that implemented nutrition-specific and nutrition-sensitive interventions to reduce stunting, mostly in a community-based setting ${ }^{9}$ in LMIC's, ${ }^{10}$ and where there were data on baseline and follow-up or end line stunting status. Studies without any components of nutrition intervention, individual randomised controlled trials (RCTs) and those in which linear growth or stunting had not been measured were excluded. The outcome of interest was changes in stunting prevalence following a programme intervention among children under 5 years of age.

The population, intervention, comparators and outcomes framework

\begin{tabular}{|c|c|c|c|}
\hline Population & Intervention & Comparison & Outcome \\
\hline $\begin{array}{l}\text { Children aged } \\
\text { between } 0 \text { and } \\
59 \text { months }\end{array}$ & $\begin{array}{l}\text { Nutrition-specific and } \\
\text { nutrition-sensitive } \\
\text { interventions implemented } \\
\text { either alone or in combination }\end{array}$ & $\begin{array}{l}\text { Programmes other } \\
\text { than nutrition } \\
\text { intervention }\end{array}$ & $\begin{array}{l}\text { Stunting } \\
\text { reduction }\end{array}$ \\
\hline
\end{tabular}

The reviewers/authors followed 'Preferred Reporting Items for Systematic Reviews and Meta-analyses (PRISMA)' checklist during this review (see online supplementary appendix 2). ${ }^{11}$ Titles and abstracts were screened by two authors independently $(\mathrm{MH}$ and $\mathrm{KABA})$. All titles and abstracts from each search were examined, matched and then the relevant articles were obtained for review (figure 2). To ensure consistency a calibration exercise was conducted. The reviewers agreed on the criteria and applied them to a sample of $20 \%$ of the retrieved studies to demonstrate adequate inter-examiner agreement ( $\kappa: 0.70-0.75$ ). The reviewers read each study independently and any disagreements were resolved through discussion or, where necessary, through consultation with a third team member (PM).

\section{Data extraction}

Two reviewers (MH and KABA) independently extracted both quantitative and qualitative data on: the number of programme components implemented, the baseline rate of stunting prevalence, the stunting prevalence following the period of the programme intervention and the rate of stunting reduction over time. They used standardised forms, checklists, note taking and annotation to compile the data from studies employing diverse interventional packages across multiple settings and geographical locations. During study selection and data extraction the reviewers were not blinded to authorship, journal of publication or the trial results.

\section{Methodological quality assessment}

The quality of the individual studies that were included was assessed by two reviewers ( $\mathrm{MH}$ and KABA) independently for both experimental (RCT) and observational (cross-sectional) studies. The possible risk of bias in RCT was assessed using the 
Figure 2 Flow diagram for the literature search.
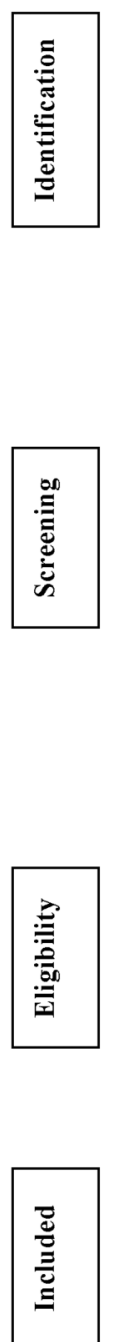

6267 titles and abstract identified through

database and screening for relevance

5015 titles and abstract excluded as

irrelevant

1141 titles and abstract further excluded as duplicates

111 papers for full text review to assess study eligibility

\begin{tabular}{|l|l|l|}
\hline $\begin{array}{l}27 \text { additional papers } \\
\text { identified for full text review } \\
\text { from reference list of } \\
\text { relevant review papers and } \\
\text { grey literature }\end{array}$ & $\rightarrow \begin{array}{l}117 \text { papers ineligible: } \\
\text { - Program did not implement intervention } \\
\text { component according to Lancet series } \\
2013 \\
- \text { Not in children }<5 \text { years } \\
\text { - Other than WHO growth standard used } \\
\text { to define and measure stunting }\end{array}$ \\
\hline
\end{tabular}

21 studies assessed eligible for inclusion

3 papers omitted

- No baseline or end line

information

- Insufficient data on outcome of interest (stunting reduction/year)

Finally 18 studies included in the systematic review
Cochrane Collaboration tool ${ }^{12}$ and quality assessment of crosssectional studies was assessed with a modified version of the Newcastle-Ottawa Scale. ${ }^{13}$ Judgments as to the possible risk of bias was rated as 'high risk' or 'low risk' for the extracted information for each of the six domains of RCT (see online supplementary appendix 3). The scale scores for observational studies ranged from 0 (lowest grade) to 7 (highest grade). Observational studies with scores at or above the median (equal to or greater than 5) were classified as high quality studies (see online supplementary appendix 4). Risk of bias across studies was assessed using the approach outlined by the 'Grading of Recommendations Assessment Development and Evaluation' (GRADE) working group. ${ }^{14}$ The quality of evidence was assessed as high, moderate and low or very low (see online supplementary appendix 5), and any disagreement was resolved by discussion or where necessary by consultation with a third author.

\section{Data analysis}

To enable comparisons to be made among the different studies, the average annual rate of reduction (AARR) was derived, as described in detail elsewhere. ${ }^{15}$ For the purpose of our investigation, we considered a programme to be effective if an AARR for stunting that was equal to or greater than the median AARR. We further characterised the individual nutrition-related components of each specific programme to determine which of the components had been implemented in the greater number of effective programmes in order to provide an indication of the consistency with which the individual components appeared to contribute to the reduction in stunting (see online supplementary appendices 6 and 7). We applied the realist approach in assessing the programme context and underlying mechanisms which might explain the programme's success in reducing stunting. ${ }^{16}$ With further discussion and critical review of the programme evaluation reports and grey literature we identified several contextual factors of probable relevance. These were themed for the different underlying contexts into broad key concepts or connections, which were considered to capture the likely mechanism(s) behind successful programme outcome (stunting reduction) (figure 3 ).

\section{Protocol and registration}

A full protocol for the study was completed by the authors prior to commencement of the study (see online supplementary appendix 8) and can be accessed at http://www.crd.york. ac.uk/PROSPERO/display_record.asp?ID=CRD42016043772. Registration no: CRD42016043772.

\section{Role of the funding source}

The funding institution had no role in the design and development, data extraction, analysis and interpretation of the data, or 
Figure 3 Realist review framework.

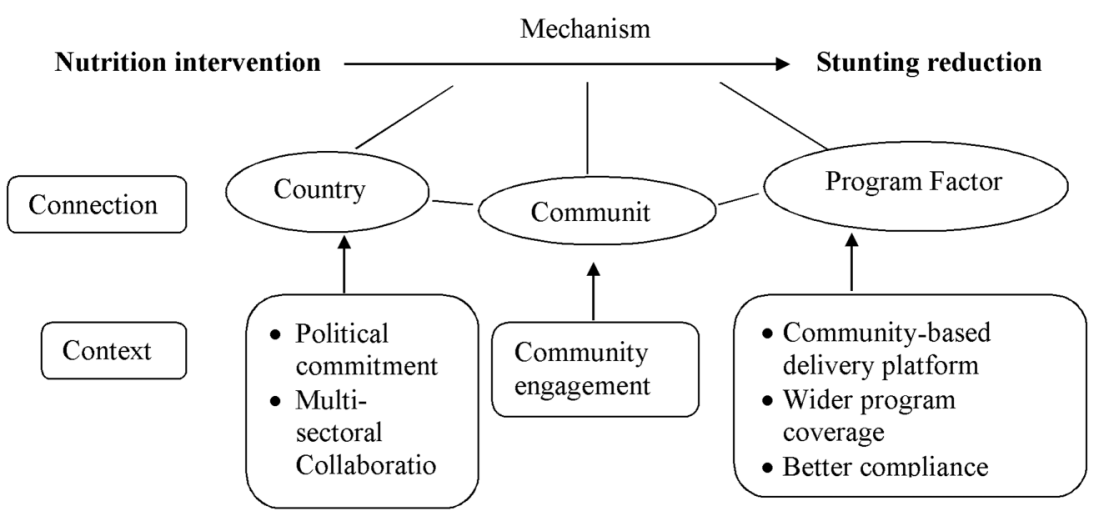

(Malawi, Niger and sub-Saharan Africa) where there was high malaria prevalence. The majority of SSN programmes were implemented in Latin American countries and targeted poor beneficiaries. The AARR in these countries varied widely (between 2.2\% and 6.7\%), even when the individual components for the intervention appeared similar (nutrition education and counselling, vitamin A supplementation, immunisation, WASH, food security and SSN). Programmes which recruited younger children (0-36 months) from poor rural households and which were implemented in areas with no other health programmes (Bangladesh, Peru and sub-Saharan Africa) reduced stunting more effectively than others (Ethiopia, Haiti, India, Malawi and Mexico) (table 1).

\section{Contextual factors behind programme outcome}

A realist approach was used to examine contextual factors which were considered to be the drivers for successful programme outcome (reduction of stunting). We identified four key concepts underpinning the connection between programme intervention and outcome. This analysis suggests that a stunting reduction programme becomes effective (AARR of stunting at least 3\%) where there is an evidence of strong political commitment, multi-sectoral collaboration between government, nongovernment, national and international organisation, active community engagement, and where the programme is delivered through community-based platforms with high coverage and compliance ( $\geq 5$ out of 7 programme). These underlying factors were clearly identified in five of seven programmes in which stunting was effectively reduced (table 2).

\section{DISCUSSION}

\section{Summary of evidence}

This systematic review identifies that in order to achieve success with interventions designed to reduce stunting in LMICs requires a combination of factors and components which together provide a suitable context. Nutrition education and counselling, GMP, immunisation, WASH, and SSN programmes were the components most frequently included in the intervention packages. The programmes appear most successful where strong political commitment and multi-sectoral collaboration between government, non-government, national and international organisations exist and where programmes are delivered through community service delivery platforms with active community engagement.

Although nutrition is necessary, interventions that focus solely on nutrition are likely to be insufficient in themselves for many of the global contexts where there is the need to reduce stunting. This review has shown that in most settings a combination supplementary appendices 6 and 7). Programmes to prevent and treat malaria were implemented only in African countries 


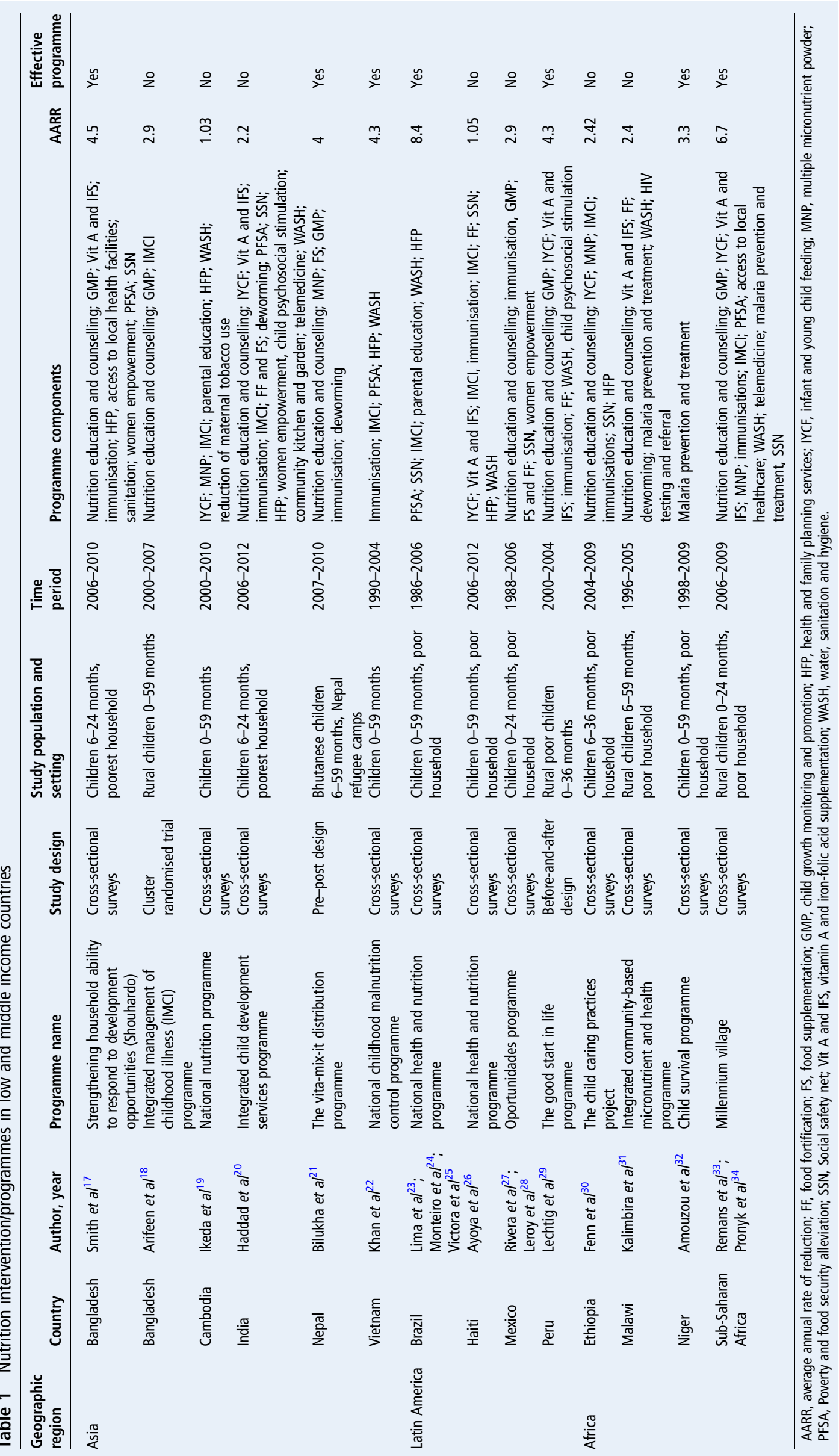

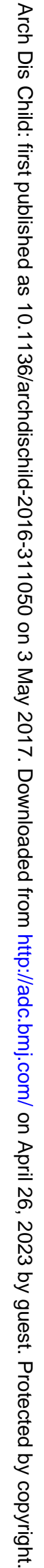


Table 2 Key concepts/connections linked to programme context and outcome

\begin{tabular}{|c|c|c|c|c|}
\hline $\begin{array}{l}\text { Key concepts/ } \\
\text { connections }\end{array}$ & $\begin{array}{l}\text { Derived from following } \\
\text { context/theme }\end{array}$ & Programme implemented in LMIC's & $\begin{array}{l}\text { No. of implemented } \\
\text { programmes }\end{array}$ & $\begin{array}{l}\text { No. of effective } \\
\text { programme ( } \geq 3 \text { AARR) }\end{array}$ \\
\hline \multirow[t]{4}{*}{ Country level } & Economic growth & Bangladesh, India, Brazil, Mexico, Cambodia & 6 & 2 \\
\hline & Strong political will & $\begin{array}{l}\text { Bangladesh, India, Nepal, Vietnam, Brazil, Haiti, Malawi, } \\
\text { Niger, Peru, sub-Saharan Africa }\end{array}$ & 10 & 6 \\
\hline & Budget allocation & India, Vietnam, Haiti, Ethiopia, Niger, sub-Saharan Africa & 6 & 3 \\
\hline & Multi-sectoral collaboration & $\begin{array}{l}\text { Bangladesh, Cambodia, India, Nepal, Vietnam, Haiti, Peru, } \\
\text { Malawi, Niger, Sub-Saharan Africa }\end{array}$ & 10 & 5 \\
\hline Community level & Community engagement & $\begin{array}{l}\text { Bangladesh, India, Nepal, Haiti, Peru, Ethiopia, Malawi, } \\
\text { Niger, Sub-Saharan Africa }\end{array}$ & 10 & 5 \\
\hline \multirow[t]{4}{*}{ Programme level } & Community-based delivery platform & $\begin{array}{l}\text { Bangladesh, India, Nepal, Haiti, Peru, Ethiopia, Malawi, } \\
\text { Niger, Sub-Saharan Africa }\end{array}$ & 10 & 5 \\
\hline & Wider programme coverage & $\begin{array}{l}\text { Bangladesh, India, Nepal, Vietnam, Brazil, Mexico, Cambodia, } \\
\text { Malawi, Niger, Sub-Saharan Africa }\end{array}$ & 9 & 5 \\
\hline & Programme compliance & $\begin{array}{l}\text { Bangladesh, India, Nepal, Vietnam, Brazil, Mexico, Cambodia, } \\
\text { Malawi, Niger, Sub-Saharan Africa }\end{array}$ & 9 & 5 \\
\hline & $\begin{array}{l}\text { Presence of other health/nutrition } \\
\text { programme }\end{array}$ & India, Nepal, Mexico, Ethiopia & 4 & 1 \\
\hline \multirow[t]{2}{*}{ Other } & Social media involvement & Bangladesh, India, Niger & 3 & 1 \\
\hline & $\begin{array}{l}\text { Environment (natural calamity, } \\
\text { disease outbreak, etc) }\end{array}$ & Bangladesh, Brazil, Ethiopia, Sub-Saharan Africa & 4 & 3 \\
\hline
\end{tabular}

AARR, average rate of reduction; LMICs, low and middle income countries.

of nutrition-specific and nutrition-sensitive approaches is needed for best effect. Even though combined interventional packages result in the greatest reductions in stunting (4.3-8.4 AARR), there is not necessarily a fixed combination of interventions that consistently demonstrate greatest benefit in all contexts. Thus, for Bangladesh, Peru and nine sub-Saharan African countries there was effective reduction of stunting with nutrition education and counselling, vitamin supplementation, immunisation, WASH, food security and SSN programmes. However, the same combinations of interventions were not similarly effective in Ethiopia, Haiti, India, Malawi or Mexico. ${ }^{20} 26283031$ The difference may be explained by the need for more secure targeting of younger children from rural household, and also the effects of other programmes or interventions in either the same or a neighbouring community. It is clear that in addition to geographical location, the organisation, administration and delivery of the intervention, as well as the population being targeted has an effect on the overall effectiveness of the intervention. However, there may be some settings in which a dominant risk factor accounts for much of the population attributable fraction of stunting and where a simple intervention can have profound impact. For example, malaria prevention and treatment has shown significant benefit in reducing stunting in regions with high malarial burden. ${ }^{32-34}$

The available evidence would not support the suggestion that any one single intervention or fixed combination of interventions is likely to achieve universal success across all settings in assuring consistent reductions in stunting. It also became clear during our analysis that when there was a seeming difference in the effect of individual components, it was likely that the context for other related intervention components had changed, altering the overall balance of possible beneficial mechanisms. The evidence supports the suggestion that programme managers and policy makers should identify and implement context specific intervention packages by addressing all three connections (country, community and programme) to achieve effective stunting reduction. At country level, Government's strong political commitment and multi-sectoral collaboration between national and international agencies provides a high level supportive framework for the formulation and coordination of appropriate policies, ${ }^{35}$ leading to the design and implementation of suitable large scale nutrition-related programmes. At the community level, community engagement enables better community-based service delivery with wider coverage and beneficiary compliance, enabling programme level interventions to achieve greater degrees of stunting reduction. ${ }^{36}$ This review supports the acknowledgement by WHO that programmes addressing the contextual factors achieved better reductions in stunting, more quickly. ${ }^{37}$

\section{Strengths and weaknesses of this review}

There are several important strengths to this analysis. The review was carried out systematically using established PRISMA and GRADE guidelines. A realist approach was adopted in evaluating underlying factors which could account for the mechanistic basis underlying programme success. This approach further helped to structure the evidence to inform recommendations on stunting reduction in different programmes employing diverse interventional packages across multiple settings and geographies. Standardised methods were used to calculate the AARR for all countries.

The review does have limitations. We included studies published in peer-reviewed journals and may have missed important unpublished data as a result of publication bias. Studies published in languages other than English were not included, which may have resulted in language bias. Finally, because many programmes combined interventions, it was not possible to attribute the level of stunting reduction directly to the effect of any single intervention.

\section{CONCLUSION AND RECOMMENDATION}

Programmes that combine nutrition-specific and nutritionsensitive interventions, particularly those with strong health access and safety net components, appear to be most effective in reducing stunting in LMICs. Given the complex nature of these diverse intervention packages, strong political commitment, multi-sectoral collaboration, community-based service delivery platforms and wider programme coverage and compliance are all likely critical components of effective stunting reduction programmes. Programme managers and policy makers should 
consider specific contextual factors in order to determine the most suitable combination of interventions while planning and implementing programmes to combat stunting.

Acknowledgements This research study was funded by core donors who provide unrestricted support to ICDDR for its operations and research. Current donors providing unrestricted support include: Government of the People's Republic of Bangladesh; Global Affairs Canada (GAC); Swedish International Development Cooperation Agency (Sida) and the Department for International Development (UK Aid). We gratefully acknowledge these donors for their support and commitment to ICDDR's research efforts. AAJ acknowledges support from NIHR Southampton Biomedical Research Centre.

Contributors $\mathrm{MH}$ and TA conceptualised the paper. MH contributed to overall coordination, collating of data sources, data analysis, tables and figures. TA and NC provided overall statistical and data analysis advice. MH, KABA and PM undertook the systematic review of published studies searches and abstraction. MH, TA, JW and AAJ provided input into the overall estimation process. All the authors reviewed and provided input to the manuscript. The authors alone are responsible for the views expressed in this article and they do not necessarily represent the views, decisions, or policies of the institutions with which they are affiliated.

\section{Competing interests None declared.}

Provenance and peer review Not commissioned; externally peer reviewed.

Open Access This is an Open Access article distributed in accordance with the Creative Commons Attribution Non Commercial (CC BY-NC 4.0) license, which permits others to distribute, remix, adapt, build upon this work non-commercially, and license their derivative works on different terms, provided the original work is properly cited and the use is non-commercial. See: http://creativecommons.org/ licenses/by-nc/4.0

\section{REFERENCES}

1 World Health Organization. WHO Child Growth Standards: Length/height-for-age, weight-for-age, weight-for-length, weight-for-height and body mass index-for-age: Methods and development. In: World Health Organization, ed. WHO Child Growth Standards. Geneva: World Health Organization, 2006.

2 Gardner JMM, Grantham-McGregor SM, Himes J, et al. Behaviour and development of stunted and Nonstunted Jamaican children. J Child Psychol Psychiatry 1999:40:819-27.

3 UNICEF. Improving child nutrition: the achievable imperative for global progress. New York, USA: United Nations Children's Fund, 2013.

4 Resnick D, Babu SC, Haggblade S, et al. Conceptualizing drivers of policy change in agriculture, nutrition, and food security: The kaleidoscope model. IFPRI Discussion Paper 1414. Washington DC: International Food Policy Research Institute (IFPRI), 2015.

5 Gillespie $S$, Haddad L, Mannar $V$, et al. The politics of reducing malnutrition: building commitment and accelerating progress. Lancet 2013;382:552-69.

6 de Onis M, Dewey KG, Borghi E, et al. The World Health Organization's global target for reducing childhood stunting by 2025: rationale and proposed actions. Matern Child Nutr 2013;9(Suppl 2):6-26.

7 McGuire S. World Health Organization. Comprehensive Implementation Plan on Maternal, Infant, and Young Child Nutrition. Geneva, Switzerland, 2014. Adv Nutr 2015;6:134-5.

8 Bhutta ZA, Das JK, Rizvi A, et al. Evidence-based interventions for improvement of maternal and child nutrition: what can be done and at what cost? Lancet 2013;382:452-77.

9 Ruel MT, Alderman H. Nutrition-sensitive interventions and programmes: how can they help to accelerate progress in improving maternal and child nutrition? Lancet 2013;382:536-51.

10 The World Bank. Country and Lending Groups 2014. http://data.worldbank.org/ about/country-and-lending-groups (accessed 15 Feb 2014).

11 Moher D, Liberati A, Tetzlaff J, et al. Preferred reporting items for systematic reviews and meta-analyses: the PRISMA statement. I Clin Epidemiol 2009;62:1006-12.

12 The Cochrane Collaboration. Part 2: General methods for Cochrane reviews. In: Higgins JP, Green S, eds. Cochrane Handbook for Systematic Reviews of Interventions. John Wiley \& Sons, Ltd, 2011:i-xxi.
13 Wells G, Shea B, O'connell D, et al. The Newcastle-Ottawa Scale (NOS) for assessing the quality of nonrandomised studies in meta-analyses. Ottawa Hospital Research Institute, 2000.

14 Guyatt G, Oxman AD, AkI EA, et al. GRADE guidelines: 1. Introduction-GRADE evidence profiles and summary of findings tables. I Clin Epidemiol 2011:64:383-94.

15 UNICEF. Technical Note: How to calculate Average Annual Rate of Reduction (AARR) of Underweight Prevalence. 2007. http://www.childinfo.org/files/Technical Note_AARR.pdf (accessed Jan 2015).

16 Pawson $R$, Greenhalgh $T$, Harvey $G$, et al. Realist review - a new method of systematic review designed for complex policy interventions. I Health Serv Res Policy 2005;10(Suppl 1):21-34.

17 Smith LC KF, Frankenberger TR, Wadud A. Admissible Evidence in the Court of Development Evaluation?: The Impact of CARE's SHOUHARDO Project on Child Stunting in Bangladesh. Brighton, UK: Institute of Development Studies, 2011.

18 Arifeen SE, Hoque DM, Akter T, et al. Effect of the Integrated Management of Childhood Illness strategy on childhood mortality and nutrition in a rural area in Bangladesh: a cluster randomised trial. Lancet 2009;374:393-403.

19 Ikeda N, Irie Y, Shibuya K. Determinants of reduced child stunting in Cambodia: analysis of pooled data from three demographic and health surveys. Bull World Health Organ 2013;91:341-9.

20 Haddad L, Nisbett N, Barnett I, et al. Maharashtra's Child Stunting Declines: What is Driving Them? Findings of a Multidisciplinary Analysis. Brighton: IDS, 2014.

21 Bilukha O, Howard C, Wilkinson C, et al. Effects of multimicronutrient home fortification on anemia and growth in Bhutanese refugee children. Food Nutr Bull 2011;32:264-76

22 Khan NC, Tuyen le D, Ngoc TX, et al. Reduction in childhood malnutrition in Vietnam from 1990 to 2004. Asia Pac J Clin Nutr 2007:16:274-8.

23 Lima AL, Silva AC, Konno SC, et al. Causes of the accelerated decline in child undernutrition in Northeastern Brazil (1986-1996-2006). Rev Saude Publica 2010:44:17-27

24 Monteiro CA, Benicio MH, Konno SC, et al. Causes for the decline in child under-nutrition in Brazil, 1996-2007. Rev Saude Publica 2009;43:35-43.

25 Victora CG, Aquino EML, Leal MdC, et al. Maternal and child health in Brazil: progress and challenges. Lancet 2011;377:1863-76.

26 Ayoya MA, Heidkamp R, Ngnie-Teta I, et al. Child malnutrition in Haiti: progress despite disasters. Glob Health Sci Pract 2013;1:389-96.

27 Rivera JA, Irizarry LM, Gonzalez-de Cossio T. Overview of the nutritional status of the Mexican population in the last two decades. Salud Publica Mex 2009;51(Suppl 4):S645-56.

28 Leroy JL, García-Guerra A, García R, et al. The oportunidades program increases the linear growth of children enrolled at young ages in Urban Mexico. J Nutr 2008;138:793-8.

29 Lechtig A, Cornale G, Ugaz ME, et al. Decreasing stunting, anemia, and vitamin A deficiency in Peru: results of the Good Start in Life Program. Food Nutr Bull 2009:30:37-48

30 Fenn B, Bulti AT, Nduna T, et al. An evaluation of an operations research project to reduce childhood stunting in a food-insecure area in Ethiopia. Public Health Nutr 2012;15:1746-54

31 Kalimbira AA, MacDonald C, Simpson JR. The impact of an integrated community-based micronutrient and health programme on stunting in Malawian preschool children. Public Health Nutr 2010;13:720-9.

32 Amouzou A, Habi O, Bensaïd K. Reduction in child mortality in Niger: a Countdown to 2015 country case study. Lancet. 2012;380:1169-78.

33 Remans R, Pronyk PM, Fanzo JC, et al. Multisector intervention to accelerate reductions in child stunting: an observational study from 9 sub-Saharan African countries. Am J Clin Nutr 2011:94:1632-42.

34 Pronyk PM, Muniz M, Nemser B, et al. The effect of an integrated multisector model for achieving the Millennium Development Goals and improving child survival in rural sub-Saharan Africa: a non-randomised controlled assessment. Lancet 2012;379:2179-88

35 Ahmed T, Hossain M, Mahfuz M, et al. Imperatives for reducing child stunting in Bangladesh. Matern Child Nutr 2016;12(Suppl 1):242-5.

36 Olney DK, Rawat R, Ruel MT. Identifying potential programs and platforms to deliver multiple micronutrient interventions. J Nutr 2012;142:178S-85S.

37 World Health Organization. WHA global nutrition targets 2025: stunting policy brief 2014. http://www.who.int/nutrition/topics/globaltargets_stunting_policybrief.pdf (accessed Feb 2015). 